\title{
A SEARCH FOR ENHANCED VERY HIGH ENERGY GAMMA-RAY EMISSION FROM THE 2013 MARCH CRAB NEBULA FLARE
}

\author{
E. Aliu ${ }^{1}$, S. Archambault ${ }^{2}$, T. Aune ${ }^{3}$, W. Benbow ${ }^{4}$, K. Berger $^{5}$, R. Bird $^{6}$, A. Bouvier $^{7}$, J. H. Buckley $^{8}$, V. Bugaev $^{8}$, \\ K. Byrum ${ }^{9}$, M. Cerruti ${ }^{4}$, X. Chen ${ }^{10,11}$, L. Ciupik ${ }^{12}$, M. P. Connolly ${ }^{13}$, W. Cui ${ }^{14}$, J. Dumm ${ }^{15}$, M. Errando ${ }^{1}$, A. Falcone ${ }^{16}$, \\ S. Federici ${ }^{11,10}$, Q. FenG ${ }^{14}$, J. P. Finley ${ }^{14}$, P. Fortin ${ }^{4}$, L. Fortson ${ }^{15}$, A. Furniss ${ }^{7}$, N. Galante ${ }^{4}$, G. H. Gillanders ${ }^{13}$, \\ S. Griffin ${ }^{2}$, S. T. Griffiths ${ }^{17}$, J. Grube ${ }^{12}$, G. GyuK ${ }^{12}$, D. Hanna ${ }^{2}$, J. Holder ${ }^{5}$, G. Hughes ${ }^{11}$, T. B. Humensky ${ }^{18}$, P. KaAret ${ }^{17}$,

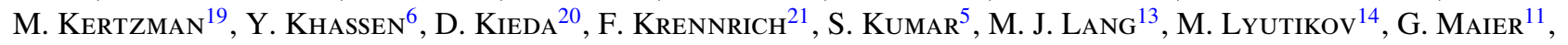 \\ S. McArthur ${ }^{22}$, A. McCann ${ }^{23}$, K. Meagher ${ }^{24}$, J. Millis ${ }^{25}$, P. Moriarty ${ }^{26}$, R. Mukherjee ${ }^{1}$, A. O’Faoláin de Bhróithe ${ }^{6}$, \\ R. A. Ong ${ }^{3}$, A. N. Otte ${ }^{24}$, N. Park ${ }^{22}$, J. S. Perkins ${ }^{27}$, M. Pohl ${ }^{10,11}$, A. Popkow ${ }^{3}$, J. QuinN ${ }^{6}$, K. RaGan ${ }^{2}$, J. Rajotte ${ }^{2}$, \\ L. C. Reyes $^{28}$, P. T. Reynolds ${ }^{29}$, G. T. Richards ${ }^{24}$, E. Roache ${ }^{4}$, G. H. Sembroski ${ }^{14}$, F. Sheidaei ${ }^{20}$, A. W. Smith ${ }^{20}$, \\ D. Staszak ${ }^{2}$, I. Telezhinsky ${ }^{10,11}$, M. Theiling ${ }^{14}$, J. V. Tucci ${ }^{14}$, J. Tyler $^{2}$, A. VARlotta $^{14}$, S. P. Wakely ${ }^{22}$, T. C. Weekes ${ }^{4}$, \\ A. Weinstein ${ }^{21}$, R. Welsing ${ }^{11}$, D. A. Williams ${ }^{7}$, A. ZajczYK ${ }^{8}$, And B. Zitzer ${ }^{9}$ \\ ${ }^{1}$ Department of Physics and Astronomy, Barnard College, Columbia University, NY 10027, USA \\ ${ }^{2}$ Physics Department, McGill University, Montreal, QC H3A 2T8, Canada \\ ${ }^{3}$ Department of Physics and Astronomy, University of California, Los Angeles, CA 90095, USA \\ ${ }^{4}$ Fred Lawrence Whipple Observatory, Harvard-Smithsonian Center for Astrophysics, Amado, AZ 85645, USA \\ ${ }^{5}$ Department of Physics and Astronomy and the Bartol Research Institute, University of Delaware, Newark, DE 19716, USA \\ ${ }^{6}$ School of Physics, University College Dublin, Belfield, Dublin 4, Ireland \\ ${ }^{7}$ Santa Cruz Institute for Particle Physics and Department of Physics, University of California, Santa Cruz, CA 95064, USA \\ ${ }^{8}$ Department of Physics, Washington University, St. Louis, MO 63130, USA \\ ${ }^{9}$ Argonne National Laboratory, 9700 S. Cass Avenue, Argonne, IL 60439, USA \\ ${ }^{10}$ Institute of Physics and Astronomy, University of Potsdam, D-14476 Potsdam-Golm, Germany \\ ${ }^{11}$ DESY, Platanenallee 6, D-15738 Zeuthen, Germany \\ ${ }^{12}$ Astronomy Department, Adler Planetarium and Astronomy Museum, Chicago, IL 60605, USA \\ ${ }^{13}$ School of Physics, National University of Ireland Galway, University Road, Galway, Ireland \\ ${ }^{14}$ Department of Physics, Purdue University, West Lafayette, IN 47907, USA \\ ${ }^{15}$ School of Physics and Astronomy, University of Minnesota, Minneapolis, MN 55455, USA \\ ${ }^{16}$ Department of Astronomy and Astrophysics, 525 Davey Lab, Pennsylvania State University, University Park, PA 16802, USA \\ ${ }^{17}$ Department of Physics and Astronomy, University of Iowa, Van Allen Hall, Iowa City, IA 52242, USA \\ ${ }^{18}$ Physics Department, Columbia University, New York, NY 10027, USA \\ ${ }^{19}$ Department of Physics and Astronomy, DePauw University, Greencastle, IN 46135-0037, USA \\ ${ }^{20}$ Department of Physics and Astronomy, University of Utah, Salt Lake City, UT 84112, USA \\ ${ }^{21}$ Department of Physics and Astronomy, Iowa State University, Ames, IA 50011, USA \\ ${ }^{22}$ Enrico Fermi Institute, University of Chicago, Chicago, IL 60637, USA \\ ${ }^{23}$ Kavli Institute for Cosmological Physics, University of Chicago, Chicago, IL 60637, USA \\ ${ }^{24}$ School of Physics and Center for Relativistic Astrophysics, Georgia Institute of Technology, 837 State Street NW, Atlanta, GA 30332-0430, USA \\ ${ }^{25}$ Department of Physics, Anderson University, 1100 East 5th Street, Anderson, IN 46012, USA \\ ${ }^{26}$ Department of Life and Physical Sciences, Galway-Mayo Institute of Technology, Dublin Road, Galway, Ireland \\ ${ }^{27}$ N.A.S.A./Goddard Space-Flight Center, Code 661, Greenbelt, MD 20771, USA \\ ${ }^{28}$ Physics Department, California Polytechnic State University, San Luis Obispo, CA 94307, USA \\ ${ }^{29}$ Department of Applied Physics and Instrumentation, Cork Institute of Technology, Bishopstown, Cork, Ireland \\ Received 2013 September 24; accepted 2013 November 7; published 2013 December 23
}

\begin{abstract}
In 2013 March, a flaring episode from the Crab Nebula lasting 2 weeks was detected by Fermi-LAT (Large Area Telescope on board the Fermi Gamma-ray Space Telescope). The Very Energetic Radiation Imaging Telescope Array System (VERITAS) provides simultaneous observations throughout this period. During the flare, Fermi-LAT detected a 20 fold increase in flux above the average synchrotron flux $>100 \mathrm{MeV}$ seen from the Crab Nebula. Simultaneous measurements with VERITAS are consistent with the non-variable long-term average Crab Nebula flux at $\mathrm{TeV}$ energies. Assuming a linear correlation between the very high energy flux change $>1 \mathrm{TeV}$ and the flux change seen in the Fermi-LAT band $>100 \mathrm{MeV}$ during the period of simultaneous observations, the linear correlation factor can be constrained to be at most $8.6 \times 10^{-3}$ with $95 \%$ confidence.
\end{abstract}

Key words: gamma rays: general - ISM: individual objects (Crab Nebula)

Online-only material: color figures

\section{INTRODUCTION}

The Crab Nebula is one of the best-studied cosmic particle accelerators. Its distance of $\sim 2 \mathrm{kpc}$ and absolute luminosity of $5 \times 10^{38} \mathrm{erg} \mathrm{s}^{-1}$ allow the study of the nebula in great detail across the entire electromagnetic spectrum. From radio to $\mathrm{GeV}$ energies, the emission is consistent with synchrotron emission of relativistic electrons (Hester 2008). However, at higher energies, the dominant emission mechanism is thought to be inverse-Compton upscattering of low-energy photons by the same population of electrons (Gould 1965; Weekes et al. 1989; de Jager \& Harding 1992; Aharonian et al. 2004).

The energy source powering the nebula is believed to be the Crab pulsar located at its center (Staelin \& Reifenstein 1968). With the pulsar as the central engine, a self-consistent magnetohydrodynamic model can be developed that explains the main features of the nebula (Rees \& Gunn 1974; Kennel \& Coroniti 1984). The discovery of flaring episodes by the AGILE 
(Tavani et al. 2011) and Large Area Telescope on board the Fermi Gamma-ray Space Telescope (Fermi-LAT; Abdo et al. 2011) teams was unexpected in this framework. The Crab Nebula flux was seen to increase by more than a factor of ten in less than a day between $100 \mathrm{MeV}$ and $1 \mathrm{GeV}$ in the most extreme of these flares.

Determining the cause of these flares is a major experimental and theoretical challenge. The observed flaring timescales of $12 \mathrm{hr}$ (Balbo et al. 2011) and $8 \mathrm{hr}$ (Buehler et al. 2012) imply that the emission region is less than $3 \times 10^{-4} \mathrm{pc}$ in diameter. This size constraint coupled with the observation that the emitted isotropic power peaks at about $1 \%$ of the pulsar spin-down power argues in favor of an emission region that moves mildly relativistically (Buehler et al. 2012; Clausen-Brown \& Lyutikov 2012; Lyutikov et al. 2012; Bednarek \& Idec 2011). As no enhancement of the pulsed emission has been observed during flares, it has been concluded that the emission region likely resides outside the corotating magnetosphere (Buehler et al. 2012; Balbo et al. 2011).

The investigation of the origin of the flares is complicated because no correlated enhancements have been observed at other wavelengths to date (Balbo et al. 2011; Striani et al. 2011, 2013; Buehler et al. 2012). Multiwavelength campaigns have been executed every time a flare has been observed since the detection of the 2010 September flare (Tavani et al. 2011). Extensive simultaneous coverage over the entire synchrotron emission from radio to X-rays did not reveal correlated activity (Horns et al. 2010; Weisskopf 2012) that could have shed light on the location of the flares due to better angular resolution at these energies.

The non-detection of correlated activity favors a monoenergetic population of relativistic electrons as the origin of the observed flares. While multiwavelength coverage has been excellent in radio, optical, and X-rays, it has been rather sparse at energies above $100 \mathrm{GeV}$, i.e., in the inverse-Compton component. No enhancement of the $\mathrm{TeV}$ emission was reported by MAGIC or the Very Energetic Radiation Imaging Telescope Array System (VERITAS) during the 2010 September flare (Mariotti 2010; Ong 2010). The ARGO-YBJ Collaboration have reported enhanced signals with a median energy of $1 \mathrm{TeV}$ from the direction of the Crab Nebula contemporaneous to GeV-band flares, although these enhancements did not reach the $5 \sigma$ level (Aielli et al. 2010; Bartoli et al. 2012; Vernetto 2013).

The electrons responsible for the flares should also upscatter soft photons in the nebula to produce $\mathrm{TeV}$ photons, which enables constraining the dynamics of the electrons. In this paper we present the most sensitive observations at $\mathrm{TeV}$ energies performed during a flare of the Crab Nebula to date. These observations with VERITAS are discussed in the context of observations with Fermi-LAT.

\section{OBSERVATIONS AND ANALYSIS}

\subsection{VERITAS}

VERITAS is an array of four $12 \mathrm{~m}$ diameter imaging atmospheric Cherenkov telescopes located at the base of Mt. Hopkins in southern Arizona, USA that observes very high energy (VHE; $E>100 \mathrm{GeV}$ ) gamma rays. Each telescope in the array has a reflector that is composed of 345 hexagonal mirror facets that focus light onto a 499-pixel photomultiplier tube camera at the focal plane with a field of view (FoV) of $\sim 3.5$. The array operates in the energy range $\sim 0.1-30 \mathrm{TeV}$ with an energy resolution of $\sim 15 \%$ at energies above $1 \mathrm{TeV}$ and an angular resolution of 0.15 (Holder et al. 2008).
VERITAS observations of the Crab Nebula in its flaring state were triggered by an automated Fermi-LAT analysis pipeline at Barnard College-Columbia University (Errando et al. 2011) on 2013 March 2, two days prior to the ATel from the FermiLAT collaboration announcing the gamma-ray flare (Ojha et al. 2013). The VERITAS data during the flare are composed of 10 nights of observations in the period MJD 56353-56366 (2013 March 2-15, henceforth referred to as the flare time window, FTW). Observations of the Crab Nebula as part of the standard observing schedule from 2012 October 13 to 2013 April 2 excluding the FTW comprise a data set on the source in its non-flaring state, which is used as a baseline with which to compare the flare data.

All VERITAS Crab observations were taken in wobble mode with an offset of 0.5 from the source position alternately in each of the four cardinal directions, so that the background can be estimated from simultaneously gathered data, and systematic effects in the background estimation cancel out (Aharonian et al. 2001; Berge et al. 2007). Observations were conducted using the full four-telescope array in a range of zenith angles $12^{\circ}-55^{\circ}$, giving a total of $10.3 \mathrm{hr}$ of live time on the source during the FTW and $17.4 \mathrm{hr}$ during the rest of the season. Two nights of flare observations (MJD 56353 and 56358) were conducted at large zenith angles, which has the effect of increasing the effective energy threshold of the array. Due to this dependence of the energy threshold, the low-energy threshold for the spectral analysis is set to a common value of $1 \mathrm{TeV}$.

The recorded images are first flat-fielded using information from nightly calibration runs taken with a pulsed UV LED (Hanna et al. 2010). The images are cleaned using a form of the picture/boundary method (Daniel 2008) and parameterized (Hillas 1985) to suppress the cosmic ray background. The shower direction is reconstructed from the data in each telescope, and a set of selection criteria is applied to reject background events (Konopelko 2001; Daniel 2008).

Energy spectra are calculated $>1 \mathrm{TeV}$ both for the FTW and the baseline observations and are shown in Figure 1. The spectra are parameterized as power laws of the form

$$
\frac{d N}{d E}=N_{0}\left(\frac{E}{1 \mathrm{TeV}}\right)^{\gamma}
$$

The baseline spectral fit gives a normalization of $N_{0}^{\text {baseline }}=$ $\left(3.48 \pm 0.14_{\text {stat. }} \pm 1.08_{\text {sys. }}\right) \times 10^{-7} \mathrm{TeV}^{-1} \mathrm{~m}^{-2} \mathrm{~s}^{-1}$ and $\gamma^{\text {baseline }}=$ $-2.65 \pm 0.04_{\text {stat. }} \pm 0.3_{\text {sys. }}$, with a $\chi^{2}$ value of 16.6 with 12 degrees of freedom (dof). The FTW spectral fit gives a normalization of $N_{0}^{\text {flare }}=\left(3.53 \pm 0.15_{\text {stat. }} \pm 1.12_{\text {sys. }}\right) \times 10^{-7} \mathrm{TeV}^{-1} \mathrm{~m}^{-2} \mathrm{~s}^{-1}$ and a spectral index $\gamma^{\text {flare }}=-2.72 \pm 0.05_{\text {sys. }} \pm 0.3_{\text {sys. }}$, with a $\chi^{2}$ value of 10.1 with 12 dof. The fit probabilities are $16 \%$ and $61 \%$, respectively. These spectral parameters are mutually consistent, implying no change of the $\mathrm{TeV}$ flux during the FTW. The systematic uncertainties on the flux normalization and spectral index are expected to vary slowly with time, and a paper containing a proper treatment of these uncertainties is currently in preparation.

\subsection{Fermi-LAT}

Fermi-LAT is a pair-conversion telescope sensitive to gammaray photons with energies between $20 \mathrm{MeV}$ and $300 \mathrm{GeV}$. It has a wide FoV of $\sim 2.5$ sr and surveys the entire sky every three hours. For a complete description of the instrument, see Atwood et al. (2009); Ackermann et al. (2012). 


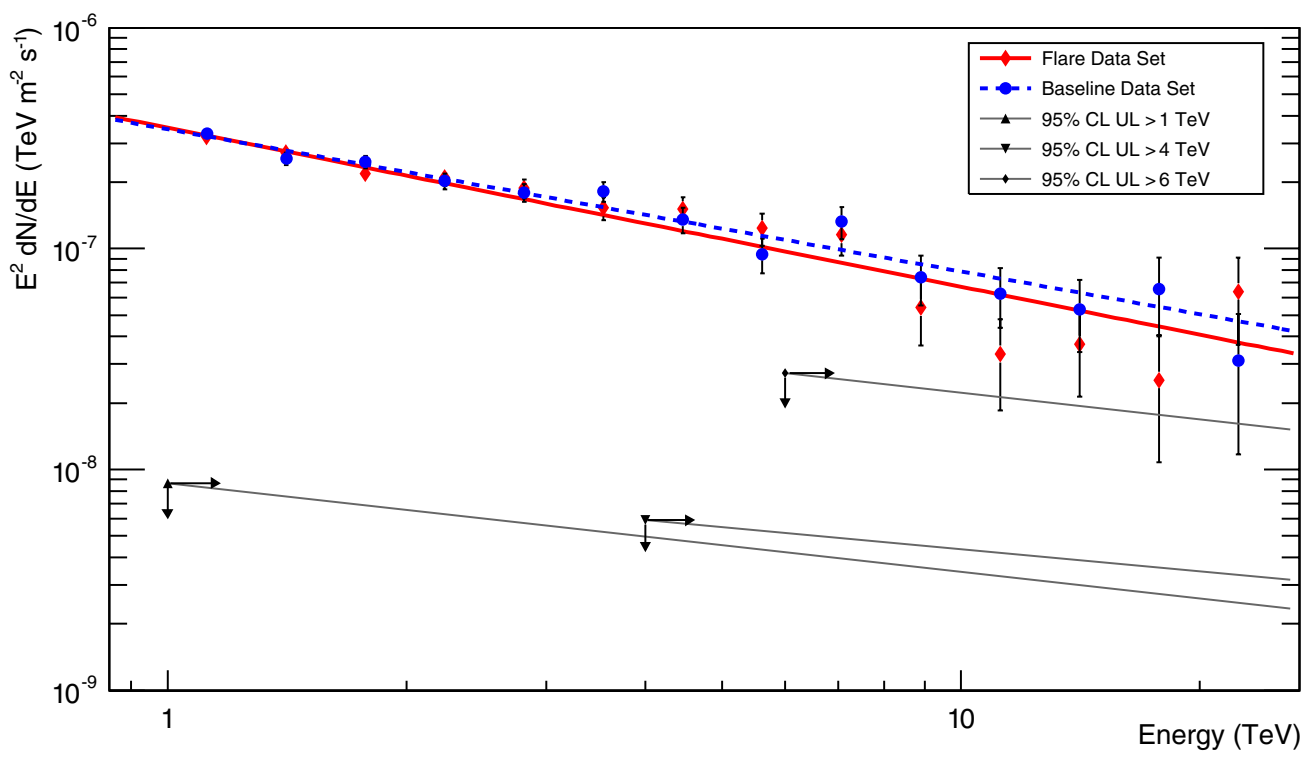

Figure 1. VHE Crab Nebula spectral energy distributions for the flare and non-flare data sets. The SEDs are fit with power-law functions (Section 2.1). From the limits on the relative flux change above $1 \mathrm{TeV}, 4 \mathrm{TeV}$, and $6 \mathrm{TeV}$ (Section 3), upper limits on an extra flux component in the flare are computed assuming a spectral index of -2.4 .

(A color version of this figure is available in the online journal.)

In order to extract spectral parameters of the Crab, the Fermi-LAT Science Tools v9r27p1 with P7V6 instrument response functions (IRFs) and the standard quality cuts described in Nolan et al. (2012) are used. Two years of "source"class events with energies between $100 \mathrm{MeV}$ and $300 \mathrm{GeV}$ collected between MJD 54832 and 55562 within $20^{\circ}$ of the $\mathrm{Crab}$ are processed with the maximum likelihood fitting routine. A model of the background is obtained in a binned likelihood analysis by fitting spectral models for all sources in the 2FGL catalog within $20^{\circ}$ of the Crab in addition to the galactic and isotropic diffuse backgrounds (gal_2yearp7v6_v0.fits, iso_p7v6source.txt). Photon arrival times are barycentered with Tempo2 (Hobbs et al. 2006) using a publicly available Jodrell Bank radio ephemeris for the Crab pulsar (Lyne et al. 1993) to allow a selection of the off-pulse phase region $0.48-0.88$. Under the assumption that emission from the pulsar is negligible in the off-pulse region, spectral parameters for the synchrotron and inverse-Compton components of the Crab Nebula are calculated. These parameters are fixed in the model to allow fitting of the pulsar spectral component after undoing the selection on pulsar phase.

The Crab Nebula synchrotron differential spectrum is parameterized as a power law (PowerLaw2 in the Fermi-LAT Science Tools) of the form

$$
\frac{d N}{d E}=\frac{F_{0}(\gamma+1) E^{\gamma}}{(300 \mathrm{GeV})^{\gamma+1}-(0.1 \mathrm{GeV})^{\gamma+1}} .
$$

The fit of the quiescent state yields a synchrotron integral flux above $100 \mathrm{MeV}$ of $F_{0}=(6.40 \pm 0.11) \times 10^{-7} \mathrm{~cm}^{-2} \mathrm{~s}^{-1}$ and photon index of $\gamma=-3.69 \pm 0.11$, which are consistent with previously published results (e.g., Buehler et al. 2012).

A similar analysis is done for the FTW. Since Fermi-LAT carried out a targeted observation of the Crab during the flare, the recommended P7V6MC IRFs and pointed mode data selection criteria are used in this analysis. ${ }^{30}$ The synchrotron

\footnotetext{
30 http://fermi.gsfc.nasa.gov/ssc/data/analysis/documentation/Cicerone/ Cicerone_Likelihood/Exposure.html
}

integral flux above $100 \mathrm{MeV}$ for the FTW is found to be $(5.30 \pm 0.13) \times 10^{-6} \mathrm{~cm}^{-2} \mathrm{~s}^{-1}$ with a harder photon index of $-3.10 \pm 0.05$. A combined spectral energy distribution (SED) showing the Fermi-LAT and VERITAS spectra is given in Figure 2.

\section{RESULTS}

A test for variability in the VERITAS FTW light curve (shown in Figure 3) is performed by fitting the light curve with a constant flux. This fit gives a flux $>1 \mathrm{TeV}$ of $(2.05 \pm 0.07) \times 10^{-7} \mathrm{~m}^{-2} \mathrm{~s}^{-1}$ with a $\chi^{2}$ value of 19.1 with 9 dof (probability $\sim 2.4 \%$ ). By fitting a light curve of data taken outside of the FTW, the Crab Nebula is detected with a baseline VHE flux $>1 \mathrm{TeV}$ of $(2.10 \pm 0.06) \times 10^{-7} \mathrm{~m}^{-2} \mathrm{~s}^{-1}$ with a $\chi^{2}$ value of 21.7 with 22 dof (probability $\sim 47.8 \%$ ). The FTW flux is thus consistent with the baseline flux and with no statistically significant variability during the flare. An analysis of a subset of the data with energies extending down to $\sim 150 \mathrm{GeV}$ was also conducted (shown in Figure 3), however no variability is revealed at these energies.

In order to test for correlated Fermi-LAT and VERITAS $(>1 \mathrm{TeV})$ flux variability in the light curves shown in Figure 3 , a publicly available implementation of the $z$-transformed discrete correlation function (ZDCF) is employed (Alexander 1997, 2013). The ZDCF method requires a minimum of 12 observations in each light curve for a statistically valid analysis, so two nights of pre-flare VERITAS Crab Nebula observations taken on MJD 56331 and 56339 (February 8 and 16, respectively) are added before the cross-correlation is performed. The zero time-lag bin reported a ZDCF correlation coefficient of

$$
\mathrm{DCF}=-0.07 \pm 0.31
$$

which is consistent with no correlation at zero lag. Results for all other time-lag bins are also consistent with no statistically significant correlation.

Relative flux changes during the FTW are calculated for VERITAS and Fermi-LAT. The $i^{\text {th }}$ relative flux change $\Delta F_{\text {rel. }}^{i}$ 


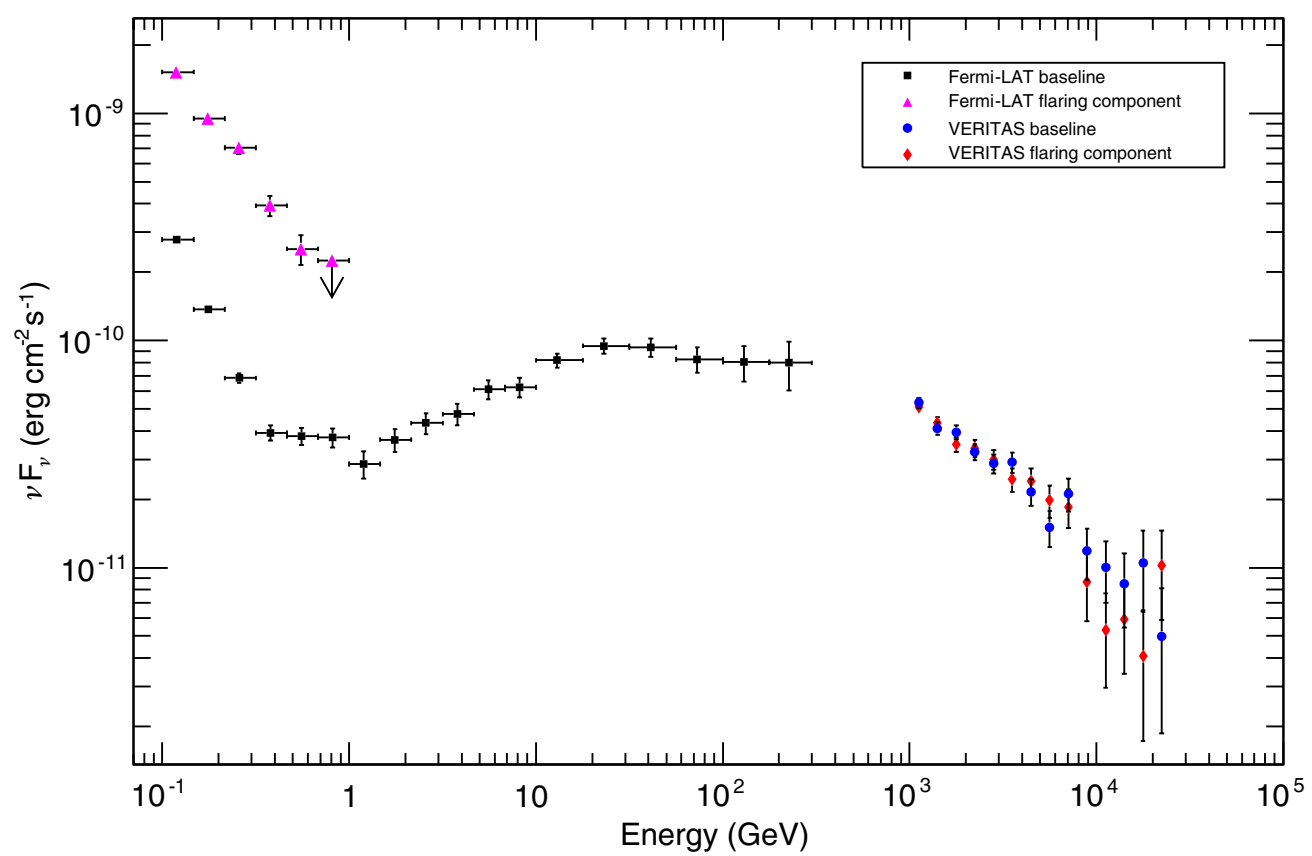

Figure 2. Combined SED of the Crab Nebula. The baseline Fermi-LAT spectrum (black squares) is averaged over $\sim 5$ yr of observations, while the baseline VHE spectrum (blue circles) includes all good data taken outside of the FTW in the 2012-2013 VERITAS observing season. The FTW VHE spectrum (red diamonds) shows no significant deviation from the baseline, while the synchrotron spectrum during this period (magenta triangles) exhibits spectral hardening. All spectral parameters given in Sections 2.1 and 2.2.

(A color version of this figure is available in the online journal.)

for both VERITAS and Fermi-LAT observations on the $i^{\text {th }}$ night is computed as

$$
\Delta F_{\text {rel. }}^{i}=\frac{F^{i}-\bar{F}}{\bar{F}}
$$

For VERITAS, $F^{i}$ is the average flux for one night. For FermiLAT, $F^{i}$ is the average flux in one $12 \mathrm{hr}$ time bin centered on midnight Arizona time (MST, 0700 UTC). $\bar{F}$ is the average non-flare flux from the nebula. The VERITAS and Fermi-LAT relative flux changes for simultaneous observations are shown in Figure 4. Averaged over the simultaneous observations in the FTW, the relative flux changes are

$$
\begin{aligned}
& \overline{\Delta F_{\text {rel. }}^{\mathrm{VTS}}}=-0.026 \pm 0.035 \quad(\text { VERITAS }>1 \mathrm{TeV}) \\
& \overline{\Delta F_{\text {rel. }}^{\mathrm{Fermi}}}=6.14 \pm 0.38 \quad(\text { Fermi-LAT }>100 \mathrm{MeV})
\end{aligned}
$$

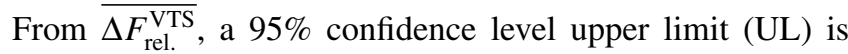
computed for an elevated VHE flux. Given the assumption of a positive and non-zero correlation of Fermi-LAT and VERITAS flux changes, a Bayesian prior is introduced in the limit calculation that is zero for negative relative flux changes and one elsewhere. This prior is equivalent to invoking the physical constraint that all of the VHE flux changes are at least zero. The UL is calculated over the Bayesian interval $\left[0, x^{\mathrm{up}}\right]$ such that

$$
\frac{\int_{0}^{x^{\text {up }}} \exp \left(-\frac{\left.\overline{\left(\Delta F_{\mathrm{rel}}^{\mathrm{VTS}}\right.}-x\right)^{2}}{2 \sigma^{2}}\right) d x}{\int_{0}^{\infty} \exp \left(-\frac{\left(\overline{\left.\Delta F_{\mathrm{rel}}^{\mathrm{VTS}}-x^{\prime}\right)^{2}}\right.}{2 \sigma^{2}}\right) d x^{\prime}}=0.95\left(x^{\mathrm{up}}>0\right),
$$

Table 1

95\% CL Bayesian Upper Limits on the VHE Relative Flux Increase During the Flare Period for Three Energy Thresholds

\begin{tabular}{lrc}
\hline \hline $\begin{array}{l}\text { Energy Band } \\
(\mathrm{TeV})\end{array}$ & $\overline{\Delta F_{\text {rel. }}^{\mathrm{VTS}}} 95 \%$ CL UL & $\begin{array}{c}95 \% \text { CL Integral UL at Threshold } \\
\left(\mathrm{TeV} \mathrm{m}^{-2} \mathrm{~s}^{-1}\right)\end{array}$ \\
\hline$>1$ & $5.3 \%$ & $8.7 \times 10^{-9}$ \\
$>4$ & $6.8 \%$ & $5.9 \times 10^{-9}$ \\
$>6$ & $37.4 \%$ & $2.7 \times 10^{-8}$ \\
\hline
\end{tabular}

where $\sigma$ is the error on $\overline{\Delta F_{\text {rel. }}^{\mathrm{VTS}}}$, and the $95 \% \mathrm{CL}$ UL is given by $x^{\text {up }}$, which is obtained by solving the equation numerically. Limits are calculated for three different energy thresholds shown in Table 1.

By adopting the assumption that the relative flux change seen by VERITAS is linearly related to that seen by Fermi-LAT during the flare:

$$
\overline{\Delta F_{\text {rel. }}^{\mathrm{VTS}}}=\alpha \overline{\Delta F_{\text {rel. }}^{\text {Fermi }}},
$$

a constraint on the linear correlation factor $\alpha$ can be calculated, which can be used to test model predictions. Taking the ratio of the $>1 \mathrm{TeV}$ UL and the average Fermi-LAT relative flux change, we find that $\alpha<8.6 \times 10^{-3}(95 \% \mathrm{CL})$ for the average of the ten nights of simultaneous observations. The constraint on $\alpha$ is also computed night-by-night, though only MJD 56358 gives the slightly better constraint of $\alpha<8.1 \times 10^{-3}$ (95\% CL).

\section{DISCUSSION}

In this paper we present observations of the Crab Nebula with VERITAS and Fermi-LAT during the 2013 March flare. The light curve and reconstructed energy spectrum between 


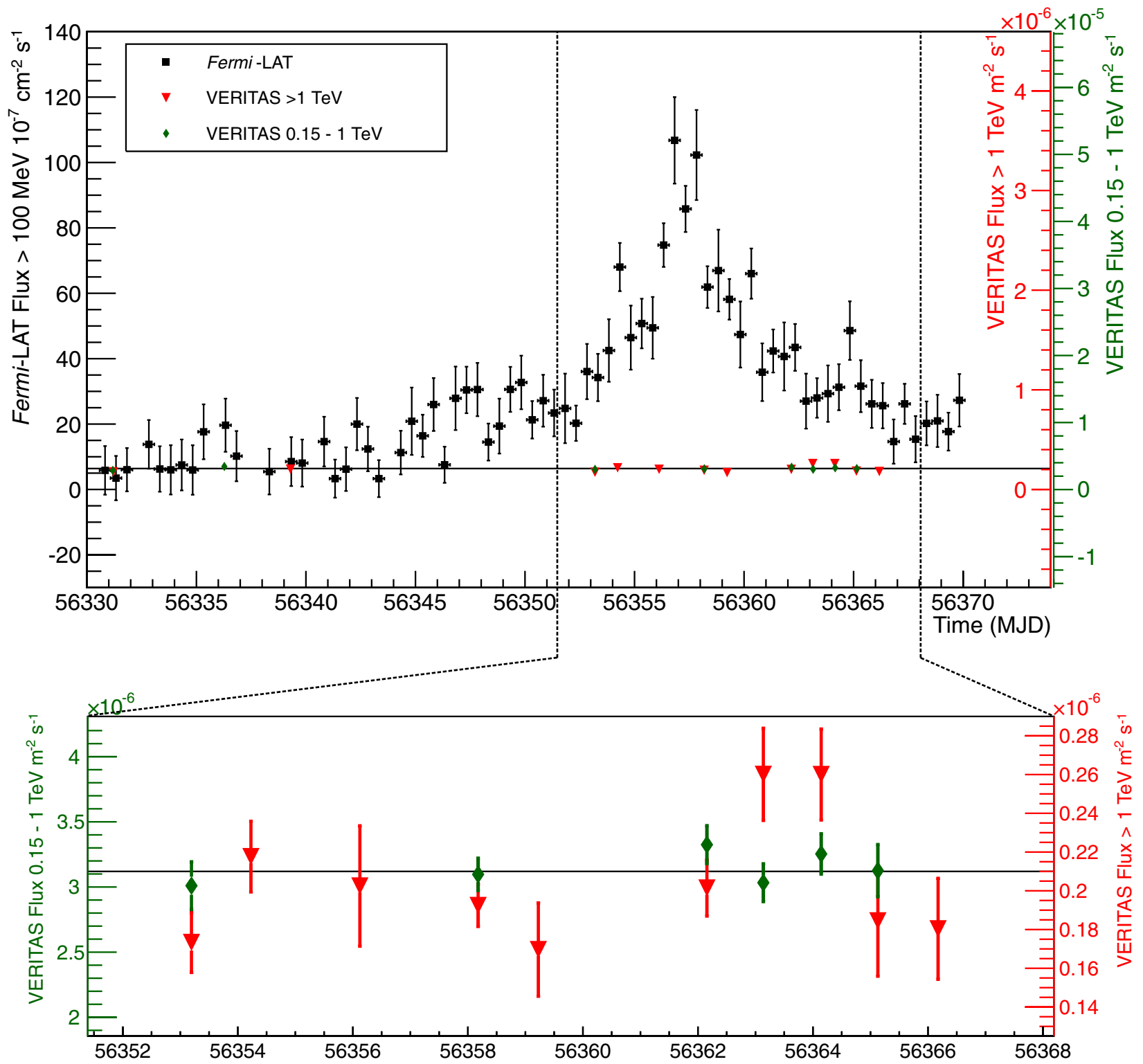

Figure 3. Fermi-LAT and VERITAS light curves for the 2013 March Crab Nebula flare. The $12 \mathrm{hr}$ binned Fermi-LAT light curve (square markers) spans MJD 56330-56370. The VERITAS light curves (triangle and diamond markers) span ten nights during the FTW where weather permitted observations. The baseline Crab Nebula synchrotron flux above $100 \mathrm{MeV}$ and average VHE flux above $0.15 \mathrm{TeV}$ and $1 \mathrm{TeV}$ are aligned and are indicated by the solid black line. The vertical scales of the three light curves have been adjusted such that the zero points and baseline fluxes are coincident.

(A color version of this figure is available in the online journal.)

$1 \mathrm{TeV}$ and $10 \mathrm{TeV}$ do not indicate any flux enhancement at $\mathrm{TeV}$ energies, while the flux above $100 \mathrm{MeV}$ was six times elevated during our observations.

Earlier flares had very hard spectra with peak energy reaching up to $\epsilon_{\text {flare }} \approx 500 \mathrm{MeV}$ (Buehler et al. 2012), though in the present flare, a peak could not be resolved in the $\mathrm{MeV}-\mathrm{GeV}$ spectrum leaving the electron spectrum unconstrained at lower energies. The synchrotron spectrum above $100 \mathrm{MeV}$ is slightly harder than for the quiescent Crab, which may reflect a separate electron population and/or an increase in the magneticfield strength in the emission zone that shifts a harder section of a curved synchrotron spectrum into the frequency band accessible with Fermi-LAT. Neglecting the weak modifications arising from the possibility of mildly relativistic bulk motion, we suggest that some excess electron acceleration took place.
From classical electrodynamics, the Lorentz factor of electrons that would emit $200 \mathrm{MeV}$ synchrotron radiation is

$$
\gamma_{\mathrm{sy}}=3 \times 10^{9}\left(\frac{B}{\mathrm{mG}}\right)^{-0.5},
$$

and their energy-loss rate and life time are

$$
\begin{aligned}
\dot{E}_{\text {sy }} & =\left(8 \times 10^{-3} \mathrm{erg} \mathrm{s}^{-1}\right)\left(\frac{B}{\mathrm{mG}}\right), \\
\tau_{\text {sy }} & =\left(3 \times 10^{5} \mathrm{~s}\right)\left(\frac{B}{\mathrm{mG}}\right)^{-1.5} .
\end{aligned}
$$

Assuming a magnetic field of $1 \mathrm{mG}$ in the emission region, similar to that deduced in Bednarek \& Idec (2011), the flare 


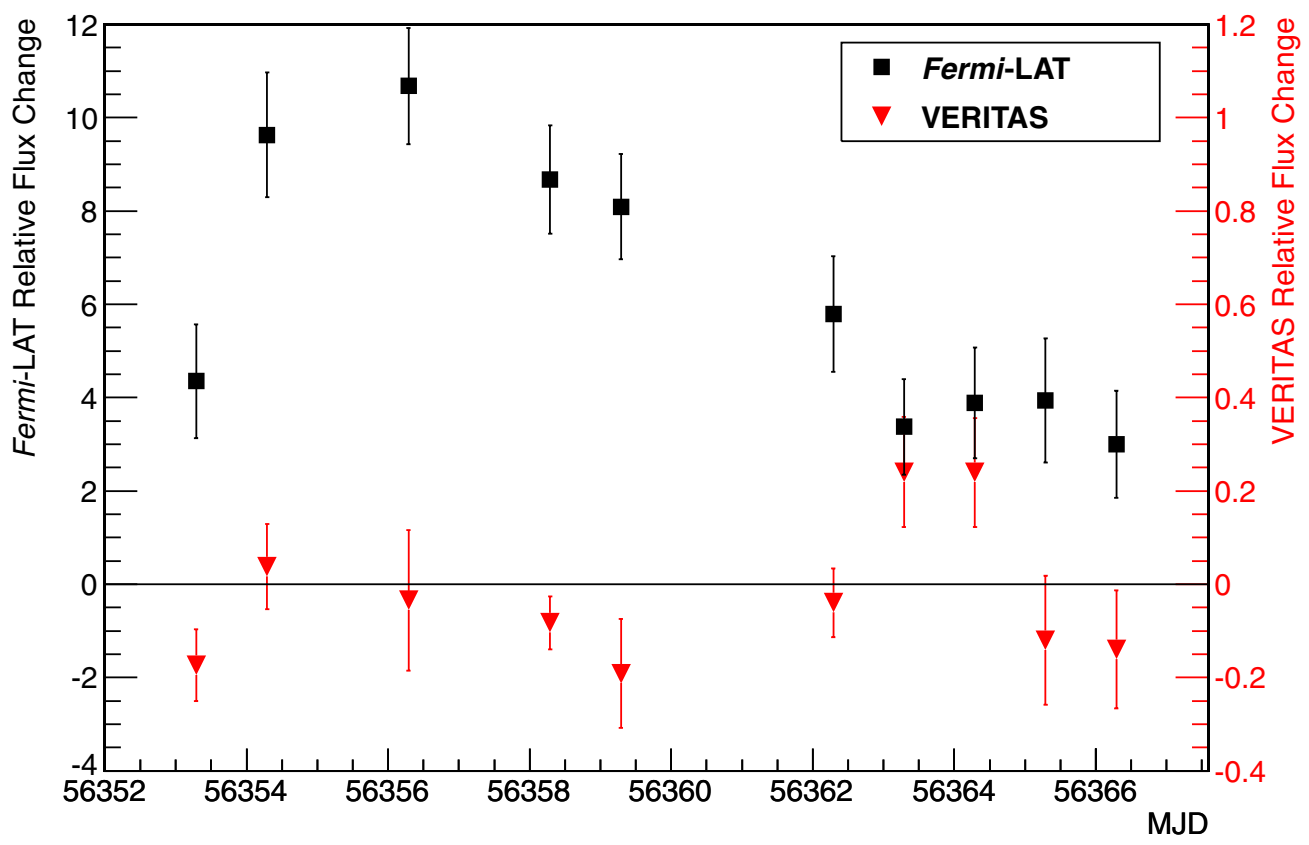

Figure 4. Relative flux changes for simultaneous Fermi-LAT (square markers) and VERITAS (triangle markers) observations during the FTW. The zero line corresponds to an observed flux equal to the average. Note that the vertical scale for the VERITAS points is a factor of ten smaller than the vertical scale for the Fermi-LAT points. (A color version of this figure is available in the online journal.)

duration $\tau_{\text {sy }}$ is on the order of a few days, which is consistent with observed flares at a few hundred MeV. If the magnetic field were significantly stronger than $1 \mathrm{mG}$, the synchrotron lifetime would become very short compared to the flare duration, and so the electron population would need to be continuously replenished to sustain the flare. Thus, the main cause of the synchrotron flare was likely the injection of a large number of excess electrons at PeV energies.

Bednarek \& Idec (2011) consider a model in which electrons are injected into the magnetic field of the pulsar wind zone and produce synchrotron gamma rays through acceleration in reconnection regions of the magnetic field. Assuming the electrons reach an equilibrium spectrum described by a differential power law with index between 3.0-3.6 and with a characteristic cutoff at $\gamma=3 \times 10^{9}$ for flares, they suggest variability above $\sim 1 \mathrm{TeV}$ of roughly $10 \%$ with more substantial changes above $\sim 10 \mathrm{TeV}$ as a result of inverse-Compton scattering. However, inverse-Compton scattering of soft photons by electrons with Lorentz factors $\sim 10^{9}$ is heavily Klein-Nishina suppressed and would provide gamma rays in the $\mathrm{PeV}$ band, beyond the reach of VERITAS. Excess electrons with Lorentz factors of $\gamma_{\mathrm{IC}} \simeq 10^{7}$ may produce a flux enhancement at $\mathrm{TeV}$ energies, but the nondetection with VERITAS poses challenges for this model and thus constrains the number of electrons with Lorentz factors of $\gamma_{\mathrm{IC}}$.

The number of electrons with Lorentz factors of $\sim 3 \times 10^{9}$ can be estimated as

$$
N_{\mathrm{e}, \mathrm{sy}}=\frac{L_{\mathrm{sy}}}{\dot{E}_{\mathrm{sy}}} \simeq 6 \times 10^{37}\left(\frac{B}{\mathrm{mG}}\right)^{-1},
$$

where $L_{\text {sy }}$ is the synchrotron luminosity at $200 \mathrm{MeV}$. To calculate the number of electrons that may inverse-Compton scatter soft (infrared, IR) photons into the TeV band, we need to know the density of low-frequency radiation in the nebula. To this end we use $L_{\text {soft }} \sim 10^{37} \mathrm{erg} \mathrm{s}^{-1}$ (Marsden et al. 1984) as the pulsar wind nebula (PWN) luminosity in IR photons, $\epsilon_{\mathrm{soft}} \sim 0.1 \mathrm{eV}$ as the photon energy, and $d_{\mathrm{PWN}} \simeq 1 \mathrm{pc}$ as the characteristic size of the Crab Nebula. The density of IR photons is then

$$
n_{\mathrm{soft}} \simeq \frac{L_{\mathrm{soft}}}{4 \pi d_{\mathrm{PWN}}^{2} c \epsilon_{\mathrm{soft}}} \simeq 20 \mathrm{~cm}^{-3} .
$$

Using the UL on an extra flux component $>1 \mathrm{TeV}$ given in Table 1 , we find that the inverse-Compton luminosity $L_{\mathrm{IC}} \lesssim$ $4 \times 10^{32} \mathrm{erg} \mathrm{s}^{-1}$. The number of electrons that upscatter photons to $\mathrm{TeV}$ energies is given by

$$
N_{\mathrm{e}, \mathrm{IC}}=\frac{L_{\mathrm{IC}}}{\sigma_{\mathrm{T}} n_{\mathrm{soft}} c \times \gamma m_{e} c^{2}},
$$

where $\sigma_{\mathrm{T}}$ is the Thomson cross-section. Ignoring the moderate Klein-Nishina suppression (the kinematic parameter $\left.4 \epsilon_{\text {soft }} \epsilon_{\gamma} /\left(m_{e}^{2} c^{4}\right) \simeq 10\right)$, the UL derived on excess TeV gamma rays corresponds to at most

$$
N_{\mathrm{e}, \mathrm{IC}}\left(\gamma \approx 10^{7}\right) \lesssim 10^{44}
$$

Assuming for ease of exposition that the spectrum of excess electrons follows a power law, $N_{e}(\gamma) \propto \gamma^{-s}$, the corresponding constraint on the spectral index is

$$
s \lesssim \frac{6.2+\log \left(\frac{B}{\mathrm{mG}}\right)}{2.5-\frac{1}{2} \log \left(\frac{B}{\mathrm{mG}}\right)},
$$

which permits $s \simeq 2.5$ for the fiducial magnetic-field strength of $1 \mathrm{mG}$. This index is harder than that assumed by Bednarek \& Idec (2011) and constrains the number of electrons that may be responsible for the Crab flare. Future observations with VERITAS or next-generation telescope arrays will likely provide more stringent constraints.

This research is supported by grants from the U.S. Department of Energy Office of Science, the U.S. National Science Foundation and the Smithsonian Institution, by NSERC in Canada, 
by Science Foundation Ireland (SFI 10/RFP/AST2748) and by STFC in the U.K. We acknowledge the excellent work of the technical support staff at the Fred Lawrence Whipple Observatory and at the collaborating institutions in the construction and operation of the instrument.

The authors are grateful to M. Mayer, E. Hays, and R. Buehler for a useful discussion of the Fermi-LAT data.

A. O'Faoláin de Bhróithe acknowledges the support of the Irish Research Council "Embark Initiative."

N.O. and G.R. gratefully acknowledge the support of a Fermi Guest Investigator grant.

\section{REFERENCES}

Abdo, A. A., Ackermann, M., Ajello, M., et al. 2011, Sci, 331, 739 Ackermann, M., Ajello, M., Albert, A., et al. 2012, ApJS, 203, 4 Aharonian, F., Akhperjanian, A., Barrio, J., et al. 2001, A\&A, 370, 112 Aharonian, F., Akhperjanian, A., Beilicke, M., et al. 2004, ApJ, 614, 897 Aielli, G., Camarri, P., Iuppa, R., et al. 2010, ATel, 2921, 1

Alexander, T. 1997, in Astronomical Time Series, ed. D. Maoz, A. Sternberg, \& E. M. Leibowitz (Astrophysics and Space Science Library, Vol. 218; Dordrecht: Kluwer), 163

Alexander, T. 2013, arXiv:1302.1508

Atwood, W. B., Abdo, A. A., Ackermann, M., et al. 2009, ApJ, 697, 1071

Balbo, M., Walter, R., Ferrigno, C., \& Bordas, P. 2011, A\&A, 527, L4

Bartoli, B., Bernardini, P., Bi, X. J., et al. 2012, ATel, 4258,

Bednarek, W., \& Idec, W. 2011, MNRAS, 414, 2229

Berge, D., Funk, S., \& Hinton, J. 2007, A\&A, 466, 1219

Buehler, R., Scargle, J. D., Blandford, R. D., et al. 2012, ApJ, 749, 26

Clausen-Brown, E., \& Lyutikov, M. 2012, MNRAS, 426, 1374

Daniel, M. K. 2008, in Proc. 30th International Cosmic Ray Conference, Vol 3, ed. R. Caballero, J. D’Olivo, \& G. Medina-Tanco (Mexico City, Mexico: Universidad Nacional Autónoma de México), 1325 de Jager, O. C., \& Harding, A. K. 1992, ApJ, 396, 161

Errando, M., Orr, M., \& Kara, E. 2011, in Proc. 32nd Int. Cosmic Ray Conf., Vol. 8, Automated Analysis of Fermi-LAT Data to Trigger Ground-based Gamma-ray Observations (Beijing: SPIRES), 135 (arXiv:1110.1036)

Gould, R. J. 1965, PhRvL, 15, 577

Hanna, D., McCann, A., McCutcheon, M., \& Nikkinen, L. 2010, NIMPA, 612,278

Hester, J. J. 2008, ARA\&A, 46, 127

Hillas, A. M. 1985, in Proc. 19th Int. Cosmic Ray Conf., Vol. 3, ed. F. C. Jones, J. Adams, \& M. Mason (La Jolla, USA: NASA Conf. Publ.), 445

Hobbs, G. B., Edwards, R. T., \& Manchester, R. N. 2006, MNRAS, 369,655

Holder, J., Acciari, V. A., Aliu, E., et al. 2008, in AIP Conf. Proc. 1085, High Energy Gamma-ray Astronomy, ed. F. A. Aharonian, W. Hofmann, \& F. Rieger (Melville, NY: AIP), 657

Horns, D., Tennant, A., Ferrigno, C., et al. 2010, ATel, 3058, 1

Kennel, C. F., \& Coroniti, F. V. 1984, ApJ, 283, 710

Konopelko, A. K. 2001, in High Energy Gamma-ray Astronomy: Int. Symp. Vol. 558, ed. F. A. Aharonian \& H. J. Völk (Heidelberg, Germany: AIP), 569

Lyne, A. G., Pritchard, R. S., \& Graham-Smith, F. 1993, MNRAS, 265, 1003

Lyutikov, M., Balsara, D., \& Matthews, C. 2012, MNRAS, 422, 3118

Mariotti, M. 2010, ATel, 2967, 1

Marsden, P. L., Gillett, F. C., Jennings, R. E., et al. 1984, ApJL, 278, L29

Nolan, P. L., Abdo, A. A., Ackermann, M., et al. 2012, ApJS, 199, 31

Ojha, R., Hays, E., Buehler, R., \& Dutka, M. 2013, ATel, 4855, 1

Ong, R. A. 2010, ATel, 2968, 1

Rees, M. J., \& Gunn, J. E. 1974, MNRAS, 167, 1

Staelin, D. H., \& Reifenstein, E. C., III 1968, Sci, 162, 1481

Striani, E., Tavani, M., Piano, G., et al. 2011, ApJL, 741, L5

Striani, E., Tavani, M., Vittorini, V., et al. 2013, ApJ, 765, 52

Tavani, M., Bulgarelli, A., Vittorini, V., et al. 2011, Sci, 331, 736

Vernetto, S. for the ARGO-YBJ Collaboration 2013, arXiv:1307.7041

Weekes, T. C., Cawley, M. F., Fegan, D. J., et al. 1989, ApJ, 342, 379

Weisskopf, M. C. 2012, arXiv:1211.7109 